\title{
Live demonstration: In-vivo imaging of neural activity with dynamic vision sensors
}

Taverni, Gemma ; Moeys, Diederik Paul ; Voigt, Fabian Friedrich ; Li, Chenghan ; Cavaco, Celso ;

Motsnyi, Vasyl ; Berry, Stewart ; Sipila, Pia ; Bello, David San Segundo ; Helmchen, Fritjof ; Delbruck, Tobi

DOI: https://doi.org/10.1109/BIOCAS.2017.8325097

Posted at the Zurich Open Repository and Archive, University of Zurich ZORA URL: https://doi.org/10.5167/uzh-168622

Conference or Workshop Item

Accepted Version

Originally published at:

Taverni, Gemma; Moeys, Diederik Paul; Voigt, Fabian Friedrich; Li, Chenghan; Cavaco, Celso; Motsnyi, Vasyl; Berry, Stewart; Sipila, Pia; Bello, David San Segundo; Helmchen, Fritjof; Delbruck, Tobi (2017). Live demonstration: In-vivo imaging of neural activity with dynamic vision sensors. In: Biomedical Circuits and Systems Conference (BioCAS), 2017, Turin, 19 October 2017 - 21 October 2017, IEEE.

DOI: https://doi.org/10.1109/BIOCAS.2017.8325097 


\title{
Live Demonstration: In-vivo Imaging of Neural Activity with Dynamic Vision Sensors
}

\author{
Gemma Taverni ${ }^{1}$, Diederik Paul Moeys ${ }^{1}$, Fabian Friedrich Voigt ${ }^{2}$, Chenghan Li ${ }^{3}$, Celso Cavaco ${ }^{4}$, Vasyl Motsnyi ${ }^{4}$, \\ Stewart Berry ${ }^{2}$, Pia Sipilä2, David San Segundo Bello ${ }^{4}$, Fritjof Helmchen ${ }^{2}$, Tobi Delbruck ${ }^{1}$ \\ ${ }^{1}$ Institute of Neuroinformatics, University of Zurich and ETH Zurich, Switzerland, ${ }^{2}$ Brain Research Institute, University of \\ Zurich, Switzerland, ${ }^{3}$ iniLabs GmbH, Zurich, Switzerland, ${ }^{4}$ IMEC research institute, Leuven, Belgium
}

\begin{abstract}
The demonstration shows the comparison of two novel Dynamic and Active Pixel Vision Sensors (DAVIS) in the context of a simulated neural imaging experiment. The first sensor, the SDAVIS, has, although a lower resolution $(188 \times 192)$ with respect to the previous generation of DAVIS sensors, 10X higher temporal contrast sensitivity. The second sensor, BSIDAVIS, combines a higher resolution $(346 \times 260)$ with a higher light sensitivity (quantum efficiency) because of its Back Side Illumination (BSI) manufacturing.
\end{abstract}

This demonstration is associated with the track Bio-Inspired and Neuromorphic Circuits and Systems. Associated paper submission identifier: 7109.

\section{DEMONSTRATION SETUP}

The demonstration compares the capability of two sensitive new generation DAVIS sensors [2] in the context of a simulated neural imaging experiment: the sensitive SDAVIS [2], and the Back Side Illuminated (BSI) BSIDAVIS. These sensors combine the regular Active Pixel Sensor (APS) capability to capture frames together with the detection of logarithmic temporal contrast in scene illumination (Dynamic Vision Sensor, DVS [3]).

The demonstration consists of the setup shown in Fig.1. A computer monitor reproduces real calcium imaging recordings obtained with a scientific sCMOS Hamamatsu Orca V2 on invitro mouse brain slices and on an in-vivo cranial window mouse. Both mice were genetically engineered to express the fast fluorescent calcium indicator GCaMP6f. Whenever the neural activity causes calcium release at the dendrites, the fluorescence of the neuron is excited.

The two sensors record in parallel the scene in real-time. Their event-based output is visible in jAER [4], the opensource JAVA-based software which processes and displays DVS events.

The higher sensitivity and sub-millisecond latency of these sensors allows them to pick up the low-contrast fluorescence emitted by the neurons, opening the possibility of the use of DAVIS sensors in the context of bioimaging.

\section{VISITORS EXPERIENCE}

Visitors can observe the different behaviours of the two DAVIS sensors in the simulated experiment presented for various recordings. Since the two sensors obtain their sensitivity from two different mechanisms (one from additional gain and one from a higher quantum efficiency), the resulting noise and signal to noise ratio performances can be visually appreciated by the visitors.

Furthermore, since of the application of these sensors is not restricted to bioimaging, visitors can interact with these newgeneration sensors directly, testing their features first-hand. Observing the low latency, high dynamic range and sensitivity allows the public to explore the DAVIS functionalities and gain insights in the working principles of event-based systems.

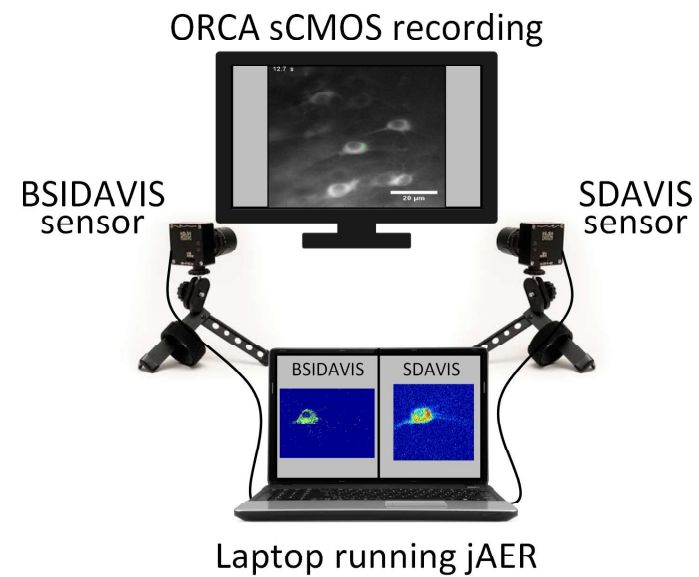

Fig.1 Demonstration setup comparing the performance of SDAVIS and BSIDAVIS in the context of neural imaging.

\section{REFERENCES}

[1] C. Brandli, R. Berner, M. Yang, S.-C. Liu, and T. Delbruck, "A 240x180 $130 \mathrm{~dB} 3$ us Latency Global Shutter Spatiotemporal Vision Sensor," IEEE J. Solid-State Circuits, vol. 49, no. 10, pp. 2333-2341, Oct. 2014

[2] D. P. Moeys and et al., "A Sensitive Dynamic and Active Pixel Vision Sensor for Color or Neural Imaging Applications," IEEE Trans. Biomed. Circuits Syst., submitted 2017.

[3] P. Lichtsteiner, C. Posch, and T. Delbruck, "A $128 \times 128120 \mathrm{~dB} 15 \mu \mathrm{s}$ Latency Asynchronous Temporal Contrast Vision Sensor," IEEE J. Solid-State Circuits, vol. 43, no. 2, pp. 566-576, 2008.

[4] "jAER Open Source Project," jAER Open Source Project, 23-Mar-2007. [Online]. Available: http://jaerproject.org. [Accessed: 23-May-2016]. 